\title{
Increasing cAMP levels of preadipocytes by cyanidin-3-glucoside treatment induces the formation of beige phenotypes in 3T3-L1 adipocytes
}

\section{Toshiya Matsukawa ${ }^{1}$, Myra O. Villareal ${ }^{2,3}$, Hideko Motojima ${ }^{3}$, and Hiroko Isoda ${ }^{2,3^{*}}$}

${ }^{1}$ Graduate School of Life and Environmental Sciences, University of Tsukuba, Tsukuba City, Ibaraki 305-8572, Japan

${ }^{2}$ Faculty of Life and Environmental Sciences, University of Tsukuba, Tsukuba City, Ibaraki 305-8572, Japan

${ }^{3}$ Alliance for Research on North Africa (ARENA), University of Tsukuba, Tsukuba City, Ibaraki 305-8572, Japan

*Corresponding author: Professor Hiroko Isoda

Faculty of Life and Environmental Sciences, University of Tsukuba

Alliance for Research on North Africa (ARENA), University of Tsukuba, Tennodai 1-1-1, Tsukuba City, Ibaraki 305-8572, Japan

E-mail: isoda.hiroko.ga@u.tsukuba.ac.jp Tel.: +81-29-853-5775; Fax: +81-29-853-5776.

Running Title: Cy3G induces formation of beige adipocyte phenotypes

Funding source: Center of Innovation Program from MEXT and JST.

Keywords: Cyanidin-3-glucoside, 3T3-L1 adipocytes, mitochondria, beige adipocyte, differentiation 


\section{Abstract}

Obesity is a serious health problem and a major risk factor for the onset of several diseases such as heart disease, diabetes, stroke, and cancer. The conversion of white adipocytes to brown-like adipocytes, also called beige or brite adipocytes, by pharmacological and dietary compounds has gained attention as an effective treatment for obesity. Cyanidin-3-glucoside (Cy3G), a polyphenolic compound contained in black soybean, blueberry and grape, has several anti-obesity effects. However, there are no reports on the role of $\mathrm{Cy} 3 \mathrm{G}$ in the induction of differentiation of preadipocytes to beige adipocytes and corresponding phenotypes. Here, the formation of beige adipocyte phenotypes following treatment with Cy3G was evaluated using 3T3-L1 adipocytes. Cy3G induced phenotypic changes to white adipocytes, such as increased multilocular lipid droplets and mitochondrial content. Additionally, the expression of mitochondrial genes (TFAM, SOD2, UCP-1, and UCP-2), UCP-1 protein, and beige adipocyte markers (CITED1 and TBX1) in 3T3-L1 adipocytes were increased by Cy3G. Furthermore, $\mathrm{Cy} 3 \mathrm{G}$ promoted preadipocyte differentiation by up-regulating of $C / E B P \beta$ through the elevation of the intracellular cAMP levels. These results indicated that Cy3G elevates the intracellular cAMP levels that induces beige adipocyte phenotypes. This is the first report on the effect of $\mathrm{Cy} 3 \mathrm{G}$ on induction of differentiation of 
preadipocytes into beige adipocyte phenotypes.

Keywords: Cyanidin-3-glucoside, 3T3-L1 adipocytes, mitochondria, beige adipocyte, differentiation 


\section{Introduction}

The number of overweight and obese individuals has been increasing annually worldwide, resulting in higher prevalence of risk factors for the onset of several diseases such as heart disease, diabetes, stroke, and cancer [1]. When energy intake exceeds energy consumption due to overeating, high-calorie intake, sedentary lifestyle, and aging, the outcome is excessive fat accumulation (increase in the number and/or size of adipocytes) [2,3]. In addition, obese individuals have decreased metabolic activity [4]. The mitochondria play a significant role in energy generation via ATP production and thermogenesis (dissipation of energy as heat). However, mitochondrial number and activity in adipocytes are decreased by increased levels of blood free fatty acids (FFA) and inflammatory cytokines in obese and diabetic patients [4]. Therefore, it is believed that increasing an individual's metabolic activity is an effective strategy for the prevention and management of medical complications associated with obesity.

The adipose tissue plays an important role in the regulation of energy balance and homeostasis. In mammals, the adipose tissue is classified into two types, white adipose tissues (WAT) and brown adipose tissues (BAT) [5]. WAT stores excess energy as triglycerides and become bigger and more numerous with weight gain [5]. WAT regulates energy homeostasis via its endocrine functions. Morphologically, 
white adipocytes contain unilocular, large lipid droplets and fewer mitochondria [5].

In obese individuals, oxygen consumption, thermogenic activity, and mitochondrial content of white adipocytes were decreased [6]. In contrast, BAT, which plays an important role in metabolism, is specialized to perform thermogenesis [7]. Morphologically, brown adipocytes contain multilocular lipid droplets, numerous mitochondria, and express uncoupling protein-1 (UCP-1) [7]. UCP-1, which is localized to the inner membrane of mitochondria, generates heat instead of ATP by diminishing the proton gradient [8]. BAT ablation or dysfunction in rodents caused obesity by decreasing energy expenditure [9]. On the other hand, enhancement of BAT function and number was effective against obesity by increasing energy expenditure [10]. Nevertheless, compared with WAT depots, BAT depots in human adults are much smaller and present in defined places, such as the neck and supraclavicular region [9]. Additionally, BAT activity and mass decline with aging [11].

Brown-like adipocytes, which contain multilocular lipid droplets and express UCP-1, appear in WAT [7]. These cells are called beige or brite (brown in white) adipocytes [7, 12]. Beige adipocytes not only have characteristics similar to brown adipocytes, such as UCP-1 expression, thermogenesis, and higher numbers of mitochondria compared to white adipocytes, but also express several beige adipocyte 
markers, such as CD137, T-box transcriptional factor (TBX1), transmembrane protein 26 (TMEM26), and Cbp/p300-interacting transactivator 1 (CITED1) [7, 8]. Brown and beige adipocytes can contribute to the regulation of whole-body energy expenditure [8]. Therefore, an increase in the number or activity level of beige adipocytes in WAT is effective for maintaining good health or prevention of obesity and other metabolic diseases [8]. It has been established that external stimulations such as exercise, chronic cold exposure, norepinephrine, irisin, and PPAR $\gamma$ agonist can induce formation of beige adipocytes [13]. Additionally, recent studies have reported that several dietary compounds, such as fucoxanthin and capsaicin, could induce formation of beige adipocytes [14].

Cyanidin-3-glucoside (Cy3G), which is a typical polyphenolic anthocyanin compound, is a pigment found in numerous colorful fruits and vegetables, such as black soybeans, blueberries, and grapes [15]. Several studies revealed that Cy3G has many positive health effects, including anti-oxidative [16] and anti-diabetic effects [17]. Furthermore, pharmacokinetic studies revealed that absorbed $\mathrm{Cy} 3 \mathrm{G}$ reaches not only the plasma but also the adipose tissues in glycosidic form [18, 19]. Therefore, it is predicted that $\mathrm{Cy} 3 \mathrm{G}$ could be an effective treatment for the prevention or treatment of obesity. We have recently reported that $\mathrm{Cy} 3 \mathrm{G}$ can induce differentiation of 
pre-adipocytes into smaller lipid droplets through the up-regulation of the peroxisome proliferator-activated receptor $\gamma$ (PPAR $\gamma)$ and CCAAT/enhancer binding protein $\alpha$ $(\mathrm{C} / \mathrm{EBP} \alpha)$ in 3T3-L1 adipocytes [17]. PPAR $\gamma$ and $\mathrm{C} / \mathrm{EBP} \alpha$ are involved in the maintenance of stable differentiation of both white and brown adipocytes [9]. Additionally, multilocular lipid droplets is one of the hallmarks of beige adipocytes [7]. However, since the effects of $\mathrm{Cy} 3 \mathrm{G}$ on the induction of beige adipocytes and its phenotypes have not yet been reported, here, we investigated the effect of $\mathrm{Cy} 3 \mathrm{G}$ on induction of beige adipocyte phenotypes using the 3T3-L1 cell line as white adipocytes cell model to gain a better understanding of its effect on obesity. 


\section{Materials \& Methods}

\subsection{Chemicals}

Cy3G, Rhodamine 123, and Rosiglitazone were purchased from Wako (Tokyo, Japan). HBSS was purchased from Gibco (NY, USA). DMEM, HEPES, Triton X-100, Radio-Immunoprecipitation Assay (RIPA) buffer, protease inhibitor cocktail, $\beta$-Actin antibody, 3-isobutyl-1-methylxanthine (IBMX), and Ro 20-1724 were purchased from Sigma (MO, USA). 3- (4,5-cimethylthiazol-2-yl)-2,5-diphenyl tetrazolium bromide (MTT) and Hoechst 33342 solution were purchased from Dojindo (Kumamoto, Japan). 2', 7'-Dichlorodihydrofluorescein diacetate (DCFH-DA) was purchased from Enzo Life Sciences (Lausen, Switzerland). UCP-1 antibody was purchased from Abcam (Cambridge, UK). PGC-1 $\alpha$ (3G6), AMPK $\alpha 1$ and phospho-AMPK $\alpha$ (Thr172) antibodies were purchased from Cell Signaling Technology (Hertfordshire, UK).

\subsection{Cell culture and differentiation}

The mouse 3T3-L1 cells (JCRB Cell Bank, Osaka, Japan) were cultured in DMEM supplemented with $10 \%$ fetal bovine serum (Gibco, USA) and 1\% penicillin (5000 $\mu \mathrm{g} / \mathrm{ml})$ - streptomycin (5000 IU/ml) (Lonza, Japan) at $37^{\circ} \mathrm{C}$ in a humidified atmosphere 
of $5 \% \mathrm{CO}_{2}$. Adipocyte differentiation and Oil Red $\mathrm{O}$ staining were performed using an Adipogenesis Assay kit (Cayman Chemical Co., Ann Arbor, USA) following the manufacturer's instructions. Briefly, for differentiation into mature adipocytes, 3T3-L1 cells were seeded at $3 \times 10^{4}$ cells $/ \mathrm{cm}^{2}$ and cultured to reach confluence. Two days post-confluence (Day 0), cells were treated with differentiation hormonal cocktail (DMEM containing DEX, IBMX and insulin) for $72 \mathrm{~h}$ (Day 3) and transferred to DMEM containing insulin that was changed every 2 days for 4 days until more than $80 \%$ of cells were differentiated (Day 7). After differentiation, cells were stained with Oil Red O. During the differentiation process (Day 0 to Day 7), 3T3-L1 cells were treated with or without Cy3G (Fig. 1 A).

\subsection{Cell proliferation (MTT assay)}

Differentiated 3T3-L1 adipocytes (Day 7) were incubated with MTT solution ( $5 \mathrm{mg} / \mathrm{ml}$ ) for $3 \mathrm{~h}$ or until formazan crystals were formed which were then dissolved by adding $10 \%$ sodium dodecyl sulfate (Wako, Japan) followed by overnight incubation (around $16 \mathrm{~h}$ ). After that, the absorbance was detected at $570 \mathrm{~nm}$ using a Powerscan HT plate reader (Dainippon Sumitomo Pharma Co, Ltd., Japan). The values were normalized to the value of the growth medium and calculated as a percentage (\%) of control. 


\subsection{Measurement of the mitochondrial content by Rhodamine 123}

Differentiated 3T3-L1 adipocytes (Day 7) were incubated with a fluorescent dye Rhodamine $123(10 \mu \mathrm{g} / \mathrm{ml})$ in $10 \mathrm{mM}$ HEPES-HBSS buffer $(\mathrm{pH} 7.4)$ for 20 min at $37^{\circ} \mathrm{C}$. Fluorescent images were obtained using a BZ-X710 All-in-One fluorescence microscope (Keyence, Osaka, Japan). To quantify the rhodamine 123 content, cells were lysed using $1 \%$ Triton X-100 and the fluorescence intensity (excitation/emission 485/528 nm) was measured using a Powerscan HT plate reader. The rhodamine 123 content was calculated using the standard curve and values were calculated as a percentage $(\%)$ of control.

\subsection{Real-time PCR analysis}

Total RNA isolation and TaqMan real-time PCR amplification reactions were performed as previously described [17]. For the quantification of gene expression, the following TaqMan probes purchased from Applied Biosystems (CA, USA) were used: $\beta$-actin (Mm00607939_s1), FABP4 (Mm00445878_m1), TFAM (Mm00447485_m1), CytC (Mm01621048_s1), PDK4 (Mm01166879_m1), SOD2 (Mm01313000_m1), UCP-1 (Mm01244861_m1), UCP-2 (Mm00627599_m1), C/EBPß (Mm00843434_s1), TBX1 
(Mm00448949_m1), CITED1 (Mm01235642_g1), PPARy (Mm01184322_m1), and

$C / E B P \alpha\left(\mathrm{Mm00514283 \_ s1).}\right.$ The PCR amplification cycles were as follows: $50^{\circ} \mathrm{C}$ for $2 \mathrm{~min}$ and $95^{\circ} \mathrm{C}$ for $10 \mathrm{~min}$, followed by 45 cycles of PCR $\left(95^{\circ} \mathrm{C}, 15 \mathrm{sec} ; 60^{\circ} \mathrm{C}, 60 \mathrm{sec}\right)$.

The mRNA levels of all genes were normalized to the $\beta$-actin level (internal control).

\subsection{Measurement of intracellular ATP level}

The intracellular ATP level in 3T3-L1 adipocytes were measured using "Cellno" ATP ASSAY reagent (TOYO Ink, Japan) according to the manufacturer's instructions. Briefly, ATP assay reagents were added to each well (100 $\mu 1 /$ well $)$ of differentiated cells (Day 7), incubated for $10 \min$ at $25^{\circ} \mathrm{C}$, and the luminescence was measured using a Powerscan HT plate reader. The values were calculated as a percentage (\%) of control.

\subsection{Measurement of intracellular ROS level}

Intracellular reactive oxygen species (ROS) level was measured using a fluorescent dye, DCFH-DA. Differentiated cells (Day 7) were cultured in serum-free DMEM containing $10 \mu \mathrm{M}$ DCFH-DA at $37^{\circ} \mathrm{C}$ for $30 \mathrm{~min}$. Then, fluorescence intensity (excitation/emission 485/528 nm) was measured using a Powerscan HT plate reader. The values were calculated as a percentage (\%) of control. 


\subsection{Western Blotting}

Total protein was extracted using RIPA buffer containing a protease inhibitor cocktail according to the manufacturer's instructions. Protein samples $(15 \mu \mathrm{g})$ were separated using $10 \%$ SDS-PAGE and transferred to a PVDF membrane (Merck Millipore, USA). Membranes were incubated with primary antibody at $4^{\circ} \mathrm{C}$ overnight, then washed and incubated with secondary antibodies, IRDye $800 \mathrm{CW}$ Donkey anti-rabbit IgG or IRDye 680LT Goat anti-mouse (LI-COR, Inc., NE, USA), at room temperature for $30 \mathrm{~min}$. The signal was detected using the OdysseyFc Imaging System (LI-COR, Inc., NE, USA). All protein quantifications were normalized to the $\beta$-Actin expression level.

\subsection{Measurement of intracellular cAMP level}

The intracellular cAMP level in 3T3-L1 cells were measured using the cAMP-Glo ${ }^{\mathrm{TM}}$ MAX assay (Promega, USA) according to the manufacturer's instructions. Briefly, 3T3-L1 cells were seeded and cultured to reach confluence in white, clear-bottom tissue culture 96 well plates (Corning, NY, USA). Two days post-confluence (Day 0), cells were cultured in serum-free DMEM containing $20 \mathrm{mM} \mathrm{MgCl}_{2}$ with or without phosphodiesterase (PDE) inhibitors (500 $\mu \mathrm{M}$ IBMX and $100 \mu \mathrm{M}$ Ro20-1724) and with 
or without Cy3G $(100 \mu \mathrm{M})$ for $30 \mathrm{~min}$. Then, the luminescence was measured using a Powerscan HT plate reader. Intracellular cAMP levels were calculated based on a standard curve and values were expressed as a percentage (\%) of control. In this assay, PDE inhibitors were used to prevent cAMP hydrolysis during the assay.

\subsection{Statistical analysis}

All the results were expressed as the mean \pm standard deviation (SD), and the statistical evaluation was performed using Student's t-test when two value sets were compared. When multiple comparisons were carried out, one way analysis of variance (ANOVA) followed by Tukey's multiple comparison test was performed using SPSS (IBM Statistics for Windows, version 22.0. IBM Corp, Armonk, NY). $P<0.05$ was considered to be statistically significant. 


\section{Results}

\subsection{Cy3G induced multilocular lipid droplets in adipocytes by promoting differentiation}

To evaluate the effect of adipocyte differentiation, 3T3-L1 preadipocytes were treated with different concentrations of $\mathrm{Cy} 3 \mathrm{G}(20,40,60,80$, and $100 \mu \mathrm{M})$ during the differentiation process (Day 0 to Day 7) (Fig. 1A). As shown in Fig. 1 B, Cy3G treatment $(20$ to $100 \mu \mathrm{M}$ ) was not cytotoxic to 3T3-L1 adipocytes. Moreover, Cy3G-treated 3T3-L1 adipocytes formed multilocular lipid droplets (Fig. 1 D). As a result of adipocyte differentiation, the expression of adipocytes markers, such as FABP4 (also known as aP2), GLUT4, and adiponectin, are reportedly up-regulated by PPAR $\gamma$ and $\mathrm{C} / \mathrm{EBP} \alpha$, an important transcriptional factor that regulates adipocyte differentiation [20]. In our previous study, we revealed that $\mathrm{Cy} 3 \mathrm{G}$ increased the expression of PPAR $\gamma$ and $C / E B P \alpha$ expression [17]. As shown in Fig. $1 \mathrm{C}, \mathrm{Cy} 3 \mathrm{G}$ treatment of $50 \mu \mathrm{M}$ and $100 \mu \mathrm{M}$ increased FABP 4 expression by 1.3 fold and 2.4 fold, respectively. Therefore, these results suggest that $\mathrm{Cy} 3 \mathrm{G}$ induced multilocular lipid droplet in adipocytes by promoting differentiation. 


\subsection{Cy3G increased the mitochondrial content and mitochondrial genes expression}

\section{through AMPK activation}

Increased mitochondrial number and the expression of mitochondrial genes, especially

UCP-1, are known markers of brown and beige adipocytes [7]. Therefore, the effect of $\mathrm{Cy} 3 \mathrm{G}$ on mitochondrial content and the expression levels of mitochondrial genes of 3T3-L1 adipocytes were evaluated. Rhodamine 123 was used as a fluorescent probe to stain mitochondria [21]. As shown in Fig. $2 \mathrm{~A}$ and B, Cy3G increased the number of mitochondria as shown by the increase in rhodamine 123 content in 3T3-L1 adipocytes to $114 \%$ and $119 \%$, respectively. The AMP-activated protein kinase (AMPK) pathway, which acts as a sensor of cellular energy status, promotes mitochondrial biogenesis, glucose uptake, and $\beta$-oxidation [22]. Mitochondrial transcriptional factor A (TFAM) is known as a key regulator of mitochondrial biogenesis, and exerts control over replication and transcription of the mitochondrial genomes [23]. As shown in Fig. 2 C, Cy3G treatments of 50 and $100 \mu \mathrm{M}$ increased phosphorylation of AMPK to 1.3 and 1.4 fold, respectively. Furthermore, the expression of TFAM and mitochondrial genes (CytC, PDK4, UCP-1,UCP-2, and SOD2) were up-regulated (Fig. 2 D). These results 
indicate that $\mathrm{Cy} 3 \mathrm{G}$ increased mitochondrial content and the expression of mitochondrial genes through AMPK activation.

\subsection{Cy3G induced similar characteristics of brown adipocytes and the expression of beige adipocyte markers}

Beige adipocytes have characteristics similar to brown adipocytes, such as UCP-1 and PGC-1 $\alpha$ expression $[7,8]$. As shown in Fig. 2 E, Cy3G increased UCP-1 and PGC-1 $\alpha$ protein expression by 2.1- and 1.7-fold, respectively. UCP-1 plays an important role in thermogenesis and decreasing the production of ROS by diminishing the proton gradient instead of ATP synthesis [24]. Additionally, Cy3G-treated 3T3-L1 adipocytes showed a decline in intracellular ROS and ATP production (Fig. $3 \mathrm{~A}$ and B). C/EBP $\beta$ is more highly expressed in brown adipocytes and regulates gain of brown adipocyte phenotypes, such as UCP-1 expression $[9,10,25]$. In the present study, $C / E B P \beta$ expression was up-regulated by Cy3G (Fig. 3 C). These results indicated that Cy3G induced similar characteristics of brown adipocyte in 3T3-L1 adipocytes. UCP-1 positive beige adipocytes selectively expressed CITED1 [26]. As shown in Fig. 3 D, Cy3G increased the expression of beige adipocyte selective markers, TBXI and CITED1. 
Therefore, these results suggest that $\mathrm{Cy} 3 \mathrm{G}$ induced beige adipocyte phenotypes in 3T3-L1 adipocytes.

\subsection{Cy3G induced the expression of beige adipocyte markers by regulating the}

\section{preadipocytes differentiation}

Chronic PPAR $\gamma$ agonist treatment has been shown to increase brown and beige adipocytes-associated genes expression in primary adipocytes differentiated from preadipocytes, it suggesting that the regulation of differentiation of preadipocytes is important step for the development of brown and beige adipocyte phenotypes in mature adipocytes [27]. In the present study, Cy3G up-regulated TBX1 and CITED1 expression in 3T3-L1 cells after 3 days of treatment (Fig. 4). CITED1 expression was especially up-regulated at Day 3 (Fig. 4). $\operatorname{PPAR} \gamma$ and $\mathrm{C} / \mathrm{EBP} \alpha$ are important transcriptional factors that regulate both white and brown adipocytes differentiation [12, 28]. During the adipocyte differentiation process, $\mathrm{C} / \mathrm{EBP} \alpha$ expression is enhanced in response to treatment with a cocktail of differentiation hormones (DEX, IBMX, and insulin) for 2-3 days [29]. As shown in Fig. 5, Cy3G increased the expression of $P P A R \gamma$ and $C / E B P \alpha$ both in the presence and in the absence of differentiation cocktail at 
3 days of induction (Day 3). Therefore, these results suggest that $\mathrm{Cy} 3 \mathrm{G}$ promoted the expression of beige adipocyte markers by regulating the preadipocytes differentiation.

\subsection{Cy3G increased $C / E B P \beta$ expression by increasing the intracellular cAMP levels through inhibition of PDE}

The transcription of $P P A R \gamma$ and $C / E B P \alpha$ is induced by $C / E B P \beta$, a widely recognized and important transcriptional factor for preadipocytes differentiation $[28,29]$. C/EBP $\beta$ expression is rapidly and transiently increased by treatment with a cocktail of differentiation hormones [28, 29]. In our present study, Cy3G significantly increased $C / E B P \beta$ expression by 1.9 fold for $1 \mathrm{~h}$ treatment and 3.2 fold for $3 \mathrm{~h}$ treatment, respectively (Fig. 6 A). Additionally, $\mathrm{Cy} 3 \mathrm{G}$ up-regulated $C / E B P \beta$ expression by 1.8 fold in the absence of the differentiation hormonal cocktail (Fig. 6 B). Intracellular cAMP levels are known to regulate the expression of C/EBP $\beta$ [30], and as shown in Fig. $6 \mathrm{C}, \mathrm{Cy} 3 \mathrm{G}$ increased the intracellular cAMP level to $138 \%$. In the absence of PDE inhibitors (IBMX and Ro 20-1724), intracellular cAMP levels were significantly lower than those of the Cy3G (PDE inhibitor +), indicating considerable cAMP hydrolysis (Fig. $6 \mathrm{C}$ ). Therefore, these results indicate that $\mathrm{Cy} 3 \mathrm{G}$ increased $C / E B P \beta$ expression 
by increasing the intracellular cAMP levels through inhibition of PDE.

\section{Discussion}

Obesity is a serious metabolic disorder that can contribute to the onset of several diseases such as diabetes, hypertension, and arteriosclerosis [1]. Therefore, the prevention and improvement of obesity is important for attaining and maintaining good health. Increased metabolic activity is effective for the prevention and improvement of obesity. Beige adipocytes are similar to brown adipocytes in terms of thermogenic activity, UCP-1 expression, and high number of mitochondria [7, 14]. Therefore, the increase in number and activity of beige adipocytes induced by pharmacological and dietary compounds has gained attention as an effective means to fight obesity [14].

Cy3G, a polyphenolic compound, is present in high amounts in black soybeans, blueberries, and grapes [15]. Pharmacokinetic studies revealed that administered Cy3G is absorbed by adipose tissue in its unchanged glycated form, clearly indicating Cy3G can reach adipose tissue through the circulation systems $[18,31]$. In our present study, we revealed that $\mathrm{Cy} 3 \mathrm{G}$ induced the adipocytes to have not only characteristics similar to brown adipocyte, such as an increase of multilocular lipid droplets, mitochondrial content, and UCP-1 expression, but also the ability to express beige 
adipocyte-associated markers in 3T3-L1 adipocytes (Fig. 1-3). 3T3-L1 adipocytes are a well-studied in vitro model of white adipocytes. The results of this study showed that Cy3G induced the differentiation of 3T3-L1 adipocytes into beige adipocyte phenotypes which suggest the possibility of improving obesity through Cy3G administration $[17,32]$.

Obese individuals have increased mitochondrial ROS production, causing the synthesis of faulty proteins, oxidized lipids, and mtDNA mutations, which are related to mitochondrial dysfunction, decreased metabolic activity, and cellular insulin sensitivity [33]. In this study, we found that Cy3G decreased the intracellular ROS level as indicated by the changes in the expression level of adipocyte markers associated with ROS production in mitochondria (Fig. 2 D and 3 A). UCP-1, a marker of brown and beige adipocytes, plays an important role in thermogenesis and decreases the ROS produced by the mitochondria [24]. UCP-2, which is a mitochondrial anion carrier present in the mitochondrial inner membrane of WAT, also controls ROS production in the mitochondria, protecting against oxidative stress [34]. Additionally, ROS scavenging enzymes, such as superoxide dismutase (SOD), is involved in the regulation of intracellular ROS levels [33]. Therefore, the results shown in Fig. $2 \mathrm{D}$ and $3 \mathrm{~A}$ suggest that Cy3G lowers the intracellular ROS production in 3T3-L1 adipocytes by 
increasing of $U C P-1, U C P-2$, and SOD2 expression. It has been established that increased ROS production induces insulin resistance, a key pathological feature of T2DM, through the activation of c-Jun N-terminal kinase and IкB kinase [35]. In our previous study, we have shown that $\mathrm{Cy} 3 \mathrm{G}$ can ameliorate $\mathrm{T} 2 \mathrm{DM}$ by increasing the insulin sensitivity of adipocytes [17]. Therefore, it is predicted that decreased the intracellular ROS production following $\mathrm{Cy} 3 \mathrm{G}$ treatment can also be attributed to Cy3G-induced activation of insulin sensitivity.

UCP-1 dissipates the proton gradient of the mitochondrial membrane to produce heat (thermogenesis) instead of ATP [8]. It has also been established that overexpression of UCP-1 in adipocytes leads to an increase in the AMP/ATP ratio and activation of AMPK [36]. Therefore, our findings, that decreased intracellular ATP production in Cy3G-treated 3T3-L1 adipocytes (Fig. 3 B) is consistent with what is known about UCP-1 activity. AMPK pathway, which is activated in response to depleted ATP levels, promotes mitochondrial biogenesis, glucose uptake, and $\beta$-oxidation [22]. In addition to increasing mitochondrial content, $\mathrm{Cy} 3 \mathrm{G}$ increased AMPK phosphorylation and the expression of TFAM (Fig. 2). TFAM is a key regulator of mitochondrial biogenesis [23]. Therefore, it is suggested that increasing of mitochondrial content is regulated by TFAM up-regulation through the AMPK 
pathway. Some AMPK activators, such as resveratrol and curcumin, can induce brown-like adipocytes $[37,38]$. Therefore, it is most likely that the activation of AMPK in response to mitochondrial uncoupling explains the increase of mitochondria content in Cy3G-treated adipocytes.

Increased mitochondrial number is one of the hallmarks of beige adipocytes [7]. Mitochondrial biogenesis and oxidative activity play a significant role in adipocyte differentiation because the adipocyte differentiation process requires a large amount of ATP during this process [39]. Therefore, with adipocyte differentiation, mitochondrial biogenesis and oxidative capacity are induced [39, 40]. Actually, PPAR $\gamma$ agonists, such as rosiglitazone and pioglitazone, increase the mitochondrial genes and proteins expression accompanied by an increasing in the rate of adipocyte differentiation [41]. In the present study, $\mathrm{Cy} 3 \mathrm{G}$ increases mitochondrial content as well as mitochondrial oxidative genes expression, such as $C y t C$ and $P D K 4$, in 3T3-L1 adipocytes (Fig. 2). These results suggest that $\mathrm{Cy} 3 \mathrm{G}$ not only increases mitochondrial biogenesis and oxidative capacity, but also promotes adipocyte differentiation.

Differentiation of preadipocytes into mature adipocytes is associated with the regulation of adipokine secretion, insulin sensitivity, and metabolic activity [20]. PPAR $\gamma$ and $\mathrm{C} / \mathrm{EBP} \alpha$ are transcription factors for numerous adipocytes-related genes and 
protein expression, and are involved in both white and brown adipocytes differentiation

$[12,20]$. Chronic PPAR $\gamma$ agonist treatment or prolonged triiodothyronine, IBMX, and rosiglitazone treatment when preadipocytes are differentiating into mature adipocytes can result to increase in brown and beige adipocytes-associated genes expression in mature adipocytes, suggesting that the regulation of differentiation of preadipocytes is important step for the development of brown and beige adipocytes phenotypes in mature adipocytes [27, 42, 43]. In this study, we observed that $\mathrm{Cy} 3 \mathrm{G}$ also promoted the expression of beige adipocyte markers by regulating preadipocytes differentiation (Fig. 4-6). Therefore, it is clear that regulating the preadipocytes differentiation may be an important step for enhancing the production of beige adipocyte phenotypes in mature adipocytes. PPAR $\gamma$ agonists, which can promote preadipocytes differentiation, induce the formation of beige and brown adipocytes phenotypes by stabilizing the PR domain-containing protein-16 (PRDM16) and increasing early B-cell factor-2 (EBF2) expression [27, 44]. Cy3G, as we have previously reported, does not have PPAR $\gamma$ agonist activity [17]. Therefore, it is predicted that $\mathrm{Cy} 3 \mathrm{G}$ induces the formation of beige adipocytes phenotypes through a different mechanism.

$\mathrm{C} / \mathrm{EBP} \beta$ is also widely recognized as an important transcriptional factor for preadipocytes differentiation, as it induces the transcription of PPAR $\gamma$ and $\mathrm{C} / \mathrm{EBP} \alpha$ [30]. 
In committed preadipocytes, $\mathrm{C} / \mathrm{EBP} \beta$ expression is very low but it is transiently enhanced by adipogenic stimulation, specifically via cAMP stimulation [30]. Therefore, increasing $C / E B P \beta$ expression and elevation of intracellular cAMP levels by Cy3G are indicators of Cy3G promoted preadipocyte differentiation (Fig. 6). The cAMP-responsive element-binding protein (CREB) is a known as central transcriptional activator of the adipocyte differentiation, are activated by cAMP. Activated CREB induces expression of $\mathrm{C} / \mathrm{EBP} \beta[45,46]$. As shown in Fig. 6, Cy3G increased intracellular cAMP levels (30 min treatment) and $C / E B P \beta$ expression ( $1 \mathrm{~h}$ treatment). Therefore, we suggest that increasing intracellular cAMP levels up-regulate $C / E B P \beta$ expression. In the present study, C/EBP $\beta$ expression was up-regulated by $\mathrm{Cy} 3 \mathrm{G}$ in both the early phase ( $1 \mathrm{~h}$ and $3 \mathrm{~h}$ ) and the mature stage (Day 7) (Fig. $3 \mathrm{C}$ and 6). $\mathrm{C} / \mathrm{EBP} \beta$ has play an important role in regulating the formation of the brown adipocyte phenotype $[10,25]$. $\mathrm{C} / \mathrm{EBP} \beta$ induces the expression of PGC-1 $\alpha$ and UCP-1 by interacting with PR domain- containing protein-16 during early differentiation and in combination with cAMP stimulation, is able to induce white preadipocyte 3T3-L1 cells to a brown-like adipocytes $[10,25,43,47]$. In this study, we found that Cy3G increased PGC-1 $\alpha$ and UCP-1 expression and intracellular cAMP levels (Fig. 2 E and 6 C) which suggests that $\mathrm{Cy} 3 \mathrm{G}$-increased cAMP levels can promote the transcription of 
beige adipocytes-related genes, therefore inducing the differentiation of white preadipocyte 3T3-L1 cells into beige adipocytes (Fig. 7). It is known that activated apoptosis signal-regulating kinase 1 (ASK1)-p38 axis in response to cAMP also induces brown and beige adipocyte function such as UCP-1 expression [48]. Therefore, we anticipate that ASK1 signalling might be involved in inducing of beige adipocyte phenotypes by Cy3G.

PDEs are key enzymes for regulating intracellular cAMP and cGMP levels. According to Merlin J et al [13], elevated intracellular cAMP levels and inhibitor of PDEs, which induce UCP-1 expression in BAT and iWAT. In the present study, Cy3G increased the intracellular cAMP level by inhibiting PDE (Fig. 6 C). Based on in vitro cell-free assay results rather than a cellular model assay, Cy3G showed PDE inhibitory effect [49]. Therefore, we suggest that Cy3G increases the intracellular cAMP levels through PDE inhibition.

In conclusion, the present study revealed that Cy3G induces the 3T3-L1 adipocytes to obtain not only similar characteristics as the brown adipocytes, such as increased multilocular lipid droplets, mitochondrial content, and UCP-1 and PGC-1 $\alpha$ expression, but also those of the beige adipocyte markers. Additionally, Cy3G 
promoted preadipocytes differentiation by up-regulating of $C / E B P \beta$ through the elevation of the intracellular cAMP levels. These results indicated that increased cAMP levels through Cy3G treatment can induce the transcription of beige adipocytes-related genes and promote white preadipocyte 3T3-L1 cells differentiation into beige adipocytes (Fig. 7). This is the first report on the effect of Cy3G on induction of differentiation of preadipocytes into beige adipocyte phenotypes. The results of our study suggest that Cy3G may be used as an effective therapeutic agent for prevention and treatment of obesity and obesity-related metabolic diseases.

\section{Conflict of interest statement}

The authors indicated no conflicts of interest.

\section{Acknowledgment}

This research is supported by the Center of Innovation Program from MEXT and JST. 


\section{References}

[1] Kopelman PG. Obesity as a medical problem. Nature 2000; 404: 635-43.

[2] Fukagawa NK, Bandini LG, Young JB. Effect of age on body composition and resting metabolic rate. Am J Physiol 1990; 259: 233-8.

[3] Hall KD, Heymsfield SB, Kemnitz JW, Klein S, Schoeller DA, Speakman JR. Energy balance and its components: implications for body weight regulation. Am J Clin Nutr 2012; 95: 989-94.

[4] Zhang L, Keung W, Samokhvalov V, Wang W, Lopaschuk GD. Role of fatty acid uptake and fatty acid $\beta$-oxidation in mediating insulin resistance in heart and skeletal muscle. Biochim Biophys Acta 2010; 1801: 1-22. 
[5] Mueller E. Understanding the variegation of fat: novel regulators of adipocyte differentiation and fat tissue biology. Biochim Biophys Acta 2014; 1842: 352-7.

[6] Flachs P, Rossmeisl M, Kuda O, Kopecky J. Stimulation of mitochondrial oxidative capacity in white fat independent of UCP1: A key to lean phenotype. Biochim Biophys Acta 2013; 1831: 986-1003.

[7] Harms M, Seale P. Brown and beige fat: development, function and therapeutic potential. Nat Med 2013; 19: 1252-63.

[8] Sidossis L, Kajimura S. Brown and beige fat in human: thermogenic adipocytes that control energy and glucose homeostasis. J Clin Invest 2015; 125: 478-86.

[9] Peirce V, Carobbio S, Vidal-Puiq A. The different shades of fat. Nature 2014; 510: 76-83.

[10] Kajimura S, Saito M. A new era in brown adipose tissue biology: Molecular control 
of brown fat development and energy homeostasis. Annu Rev Physiol 2014; 76: 225-49.

[11] Pfannenberg C, Werner MK, Ripkens S, Stef I, Deckert A, Schmadl M et al. Impact of age on the relationships of brown adipose tissue with sex and adiposity in humans. Diabetes 2010; 59: 1789-93.

[12] Giralt M, Villarroya F. White, brown, beige/brite: different adipose cells for different functions? Endocrinology 2013; 154: 2992-3000.

[13] Merlin J, Evans BA, Dehvari N, Sato M, Bengtsson T, Hutchinson DS. Could burning fat start with a brite spark? Pharmacological and nutritional ways to promote thermogenesis. Mol Nutr Food Red 2016; 60: 18-42.

[14] Bonet ML, Oliver P, Palou A. Pharmacological and nutritional agents promoting browning of white adipose tissue. Biochim Biophys Acta 2013; 1831: 969-85.

[15] Wu X, Beecher GR, Holden JM, Haytowitz DB, Gebhardt SE, Prior RL. Concentrations of anthocyanins in common foods in the United States and estimation of 
normal consumption. J Agric Food Chem 2006; 54: 4069-75.

[16] Tsuda T, Horio F, Osawa T. Dietary cyanidin 3-O- $\beta$-D-glucoside increases ex vivo oxidation resistance of serum in rats. Lipids 1998; 33: 583-8.

[17] Matsukawa T, Inaguma T, Han J, Villareal MO, Isoda H. Cyanidin-3-glucoside derived from black soybeans ameliorate type 2 diabetes through the induction of differentiation of preadipocytes into smaller and insulin-sensitive adipocytes. J Nutr Biochem 2015; 26: 860-7.

[18] Cao G, Muccitelli HU, Sánchez-Moreno C, Prior RL. Anthocyanins are absorbed in glycated forms in elderly women: a pharmacokinetic study. Am J Clin Nutr 2001; 73: 920-6.

[19] Felgines C, Texier O, Garcin P, Besson C, Lamaison JL, Scalbert A. Tissue distribution of anthocyanins in rats fed a blackberry anthocyanin-enriched diet. Mol Nutr Food Res 2009; 53: 1098-103. 
[20] Cristancho AG, Lazar MA. Forming function fat: a growing understanding of adipocyte differentiation. Nat Rev Mol Cell Biol 2011; 12: 722-34.

[21] Johnson LV, Walsh ML, Chen LB. Localization of mitochondria in living cells with rhodamine 123. Proc Natl Acad Sci U S A 1980; 779: 90-4.

[22] Hardie DG, Ross FA, Hawley SA. AMPK: a nutrient and energy sensor that maintains energy homeostasis. Nat Rev Mol Cell Biol 2012; 13: 251-62.

[23] Butow RA, Bahassi EM. Adaptive thermogenesis: Orchestrating mitochondrial biogenesis. Curr Biol 1999; 9: 767-9.

[24] Brondani LA, Assmann TS, Duarte GC, Gross JL, Canani LH, Crispim D. The role of the uncoupling protein 1 (UCP1) on the development of obesity and type 2 diabetes mellitus. Arg Bras Endocrinol Metabol 2012; 56: 215-25.

[25] Fu J, Li X, Zhang Y, Mao Y, Wang A, Wang X et al. Molecular pathways regulating the formation of brown-like adipocytes in white adipose tissue. Diabetes Metab Res Rev 
$2015 ; 31: 433-52$.

[26] Sharp LZ, Shinoda K, Ohno H, Scheel DW, Tomoda E, Ruiz L et al. Human BAT possesses molecular signatures that resemble beige/brite cells. PLoS One 2012; 7 : e49452.

[27] Ohno H, Shinoda K, Spieqelman BM, Kajimura S. PPAR $\gamma$ agonists Induces a White-to-Brown Fat Conversion through Stabilization of PRDM16 Protein. Cell Metab 2012; 15: 395-404.

[28] Farmer SR. Transcriptional control of adipocyte formation. Cell Metab 2006; 4: 263-73.

[29] Darlington GJ, Ross SE, MacDougald OA. The role of C/EBP Genes in Adipocyte Differentiation. J Biol Chem 1998; 273: 30057-60.

[30] Siersbaek R, Nielsen R, Mandrup S. Transcriptional networks and chromatin remodeling controlling adipogenesis. Trends Endocrinol Metab 2012; 23: 56-64. 
[31] Miyazawa T, Nakagawa K, Kudo M, Muraishi K, Someya K. Direct Intestinal Absorption of Red Fruit Anthocyanins, Cyanidin-3-glucoside and Cyanidin-3,5-diglucoside, into Rats and Humans. J Agric Food Chem 1999; 47: 1083-91.

[32] Guo H, Xia M, Zou T, Ling W, Zhong R, Zhang W et al. Cyanidin 3-glucoside attenuates obesity-associated insulin resistance and hepatic steatosis in high-fat diet-fed and $d b / d b$ mice via the transcription factor FoxO1. J Nutr Biochem. 2012; 23: 349-60.

[33] Sivitz WI, Yorek MA. Mitochondrial Dysfunction in Diabetes: From Molecular Mechanisms to Functional Significance and Therapeutic Opportunities. Antioxid Redox Signal 2010; 12: 537-77.

[34] Chevilloate E, Giralt M, Miroux B, Ricquier D, Villarroya F. Uncoupling protein-2 controls adiponectin gene expression in adipose tissue through the modulation of reactive oxygen species production. Diabetes 2007; 56: 1042-50. 
[35] Tiganis T. Reactive oxygen species and insulin resistance: the good, the bad and the ugly. Trends Pharmacol Sci 2011; 32: 82-93.

[36] Daval M, Foufelle F, Ferré P. Functions of AMP-activated protein kinase in adipose tissue. J Physiol 2006; 574: 55-62.

[37] Wang S, Liang X, Yang Q, Fu X, Roqers CJ, Zhu M et al. Resveratrol induces brown-like adipocyte formation in white fat through activation of AMP-activated protein kinase (AMPK) $\alpha 1$. IntJ Obes (Lond) 2015; 39: 967-76.

[38] Lone J, Choi JH, Kim SW, Yun JW. Curcumin induces brown fat-like phenotype in 3T3-L1 and primary white adipocytes. J Nutr Biochem 2016; 27: 193-202.

[39] De Pauw A, Tejerina S, Raes M, Keijer J, Arnould T. Mitochondrial (Dys) function in Adipocyte (De) differentiation and Systemic Metabolic Alteration. Am J Pathol 2009; 175: 927-39.

[40] Lu R, Ji H, Chang ZG, Su SS, Yang GS. Mitochondrial development and the 
influence of its dysfunction during rat adipocytes differentiation. Mol Biol Rep 2010; 37: $2173-82$.

[41] Wilson-Fritch L, Burkart A, Bell G, Mendelson K, Leszyk J, Nicoloro S et al. Mitochondrial biogenesis and remodeling during adipogenesis and in response to the insulin sensitizer rosiglitazone. Mol Cell Biol 2003; 23: 1085-94.

[42] Asano H, Kanamori Y, Higurashi S, Nara T, Kato K, Matsui T et al. Induction of beige-like adipocytes in 3T3-L1 adipocytes. J Vet Med Sci 2014; 76: 57-64.

[43] Karamanlidis G, Karamitri A, Docherty K, Hazlerigg DG, Lomax MA. C/EBP $\beta$ reprograms white 3T3-L1 preadipocytes to a brown adipocyte pattern of gene expression. J Biol Chem 2007; 282. 24660-9.

[44] Stine RR, Shapira SN, Lim HW, Ishibashi J, Harms M, Won KJ et al. EBF2 promotes the recruitment of beige adipocytes in white adipose tissue. Mol Metab. 2015;

5: $57-65$. 
[45] Fox KE, Fankell DM, Erickson PF, Majka SM, Crossno JT Jr, Klemm DJ.

Depletion of cAMP-response element-binding protein/ATF1 inhibits adipogenic conversion of 3T3-L1 cells ectopically expressing CCAAT/enhancer-binding protein (C/EBP) alpha, C/EBP beta, or PPAR gamma 2. J Biol Chem. 2006; 281: 40341-53.

[46] Petersen RK, Madsen L, Pedersen LM, Hallenborg P, Hagland H, Viste K et al. Cyclic AMP (cAMP)-mediated stimulation of adipocyte differentiation requires the synergistic action of Epac- and cAMP-dependent protein kinase-dependent processes. Mol Cell Biol. 2008; 28: 3804-16.

[47] Chi J, Cohen P. The multifaceted roles of PRDM16: Adipose Biology and beyond. Trends Endocrinol Metab 2016; 27: 11-23.

[48] Hattori K, Naguro I, Okabe K, Funatsu T, Futami S, Takeda K et al. ASK1 signalling regulates brown and beige adipocyte function. Nat Commun 2016; 11158: doi: 10.1038/ncomms 11158 .

[49] Dallas C, Gerbi A, Tenca G, Juchaux F, Bernard FX. Lipolytic effect of a 
polyphenolic citrus dry extract of grapefruit, orange (SINETROL) in human body fat adipocytes. Mechanism of action by inhibition of cAMP-phosphodiesterase (PDE). Phytomedicine 2008; 15: 783-92.

\section{Figure legends}

Figure 1. Effect of Cy3G on lipid droplet morphology and adipocyte differentiation

\section{in 3T3-L1 adipocytes.}

Experimental procedure of differentiation of 3T3-L1 adipocytes. During the differentiation process (Day 0 to Day 7), 3T3-L1 cells were treated with or without 20 to $100 \mu \mathrm{M} \mathrm{Cy} 3 \mathrm{G}$ (A). After differentiation, cell viability (B), FABP4 mRNA levels (C), and lipid droplet morphology (D) were evaluated. (B) Cell viability was determined by MTT assay. Values are expressed as a percentage (\%) of the control. (C) FABP4 mRNA levels were normalized to $\beta$-actin expression levels. (B and C) Results are expressed as the mean $\pm \mathrm{SD}$ of triplicate experiments. $* P<0.05$, ** 
Differentiated 3T3-L1 adipocytes were stained by Oil Red O. Photographs are on the Control (Left) and $100 \mu \mathrm{M}$ Cy3G-treated 3T3-L1 adipocytes (Right). Scale bar indicates $50 \mu \mathrm{m}$.

Figure 2. Effect of Cy3G on mitochondrial content and the expression of mitochondrial genes and UCP-1 in 3T3-L1 adipocytes.

3T3-L1 preadipocytes were treated with or without 50 and $100 \mu \mathrm{M} \mathrm{Cy3G}$ during the differentiation process for 7 days. After differentiation, mitochondrial content (A, B), phosphorylation of AMPK (C), mitochondrial gene (TFAM, CytC, PDK4, SOD2, UCP-1, and $U C P-2$ ) expression (D), and UCP-1 and PGC-1 $\alpha$ protein expression (E) in 3T3-L1 adipocytes were evaluated. (A, B) Mitochondria were stained by a mitochondrial specific dye, Rhodamine 123. (A) Photographs are on the Control (Left) and $100 \mu \mathrm{M}$ Cy3G-treated 3T3-L1 adipocytes (Right). Rhodamine 123 (mitochondria) is green and Hoechst 33342 (nuclei) is blue. Scale bar indicates $100 \mu \mathrm{m}$. (B) To quantify the rhodamine 123 content, cells were lysed using 1\% Triton X-100 and fluorescence intensity $(485 \mathrm{~nm} / 528 \mathrm{~nm})$ was measured. Values were expressed as a percentage (\%) of the control. ( $\mathrm{C}$ and $\mathrm{E})$ Protein expression levels were normalized to the $\beta$-actin 


\begin{abstract}
expression levels. (D) Expression levels of mRNA were normalized to the $\beta$-actin expression level. (B and E) Rosiglitazone $(1 \mu \mathrm{M})$ is used as a positive control.
\end{abstract} Results are expressed as the mean $\pm \mathrm{SD}$ of triplicate experiments. $* P<0.05$, ** $P<0.01$ indicates the mean value is significantly different from that of the control.

Figure 3. Effect of Cy3G on intracellular ROS, ATP production, and the expression of beige adipocyte markers in 3T3-L1 adipocytes.

3T3-L1 preadipocytes were treated with or without 50 and $100 \mu \mathrm{M} \mathrm{Cy} 3 \mathrm{G}$ during the differentiation process for 7 days. After differentiation, intracellular ROS production (A), intracellular ATP production (B), and gene expression of $C / E B P \beta$ (C) and beige adipocyte markers (D) in 3T3-L1 adipocytes were evaluated. (A and B) Values were expressed as a percentage (\%) of the control. (C and D) Values were normalized to the $\beta$-actin expression levels. Results are expressed as the mean $\pm \mathrm{SD}$ of triplicate experiments. $* P<0.05, * * P<0.01$ indicates the mean value is significantly different from that of the control.

Figure 4. Time dependent effect of Cy3G on TBX1 and CITED1 expression in 3T3-L1 cells. 
3T3-L1 preadipocytes were treated with or without 50 and $100 \mu \mathrm{M} \mathrm{Cy} 3 \mathrm{G}$ during the differentiation process for 3, 5, and 7 days. After treatment, the gene expression of TBX1 (A) and CITED1 (B) in 3T3-L1 cells were evaluated using real-time PCR. (A and B) Values were normalized to the $\beta$-actin expression levels and expressed relative to the undifferentiated 3T3-L1 cells. Results are expressed as the mean \pm SD of triplicate experiments. $* P<0.05, * * P<0.01$ indicates the mean value is significantly different from that of the control.

\section{Figure 5. Effect of Cy3G on $P P A R y$ and $C / E B P \alpha$ expression in 3T3-L1 cells.}

3T3-L1 preadipocytes (Day 0) were treated with or without Cy3G $(100 \mu \mathrm{M})$ in the presence of the differentiation hormonal cocktail (A) or the absence of the differentiation hormonal cocktail (B) for 3 days. And then, gene expression of levels of PPAR $\gamma$ and $C / E B P \alpha$ were evaluated using real-time PCR and normalized to the $\beta$-actin expression levels. Results are expressed as the mean $\pm \mathrm{SD}$ of triplicate experiments. $* * P<0.01$ indicate the mean value that is significantly different from that of each control groups.

Figure 6. Effect of $\mathrm{Cy3G}$ on early phase of adipocyte differentiation in 3T3-L1 
cells.

(A) 3T3-L1 preadipocytes (Day 0) were treated with or without Cy3G $(100 \mu \mathrm{M})$ in the presence of a differentiation hormonal cocktail for 1-3 h. (B) 3T3-L1 preadipocytes were treated with or without $100 \mu \mathrm{M} \mathrm{Cy} 3 \mathrm{G}$ in the absence of the differentiation hormonal cocktail for $1 \mathrm{~h}$. Expression levels of $C / E B P \beta$ mRNA were evaluated and normalized to the $\beta$-actin expression levels. $* * P<0.01$ indicates significant difference compared to the control. (C) 3T3-L1 preadipocytes were treated with or without Cy3G $(100 \mu \mathrm{M})$ and with or without phosphodiesterase (PDE) inhibitor (500 $\mu \mathrm{M}$ IBMX and $100 \mu \mathrm{M}$ Ro20-1724) for $30 \mathrm{~min}$. After that, the intracellular cAMP level was measured. Values were calculated using the standard curve, then quantified and expressed as a percentage (\%) of the control. Results are expressed as the mean \pm SD of triplicate experiments. $* * P<0.01$ indicates significant difference compared to the Control (PDE inhibitor + ). \#\# $P<0.01$ indicates significant difference compared to the Cy3G (PDE inhibitor +). Multiple comparisons were carried out using ANOVA followed by Tukey's multiple comparison test.

Figure 7. Suggested pathway for cyanidin-3-glucoside induced beige adipocyte phenotypes by promoting adipocyte differentiation in 3T3-L1 adipocytes 
Cy3G induced beige adipocytes phenotypes, such as increased mitochondrial content and the expression of mitochondrial genes, UCP-1 expression, and beige adipocytes markers by promoting adipocyte differentiation through $C / E B P \beta$ up-regulation. This $C / E B P \beta$ up-regulation was induced by increasing the intracellular cAMP level. 
A

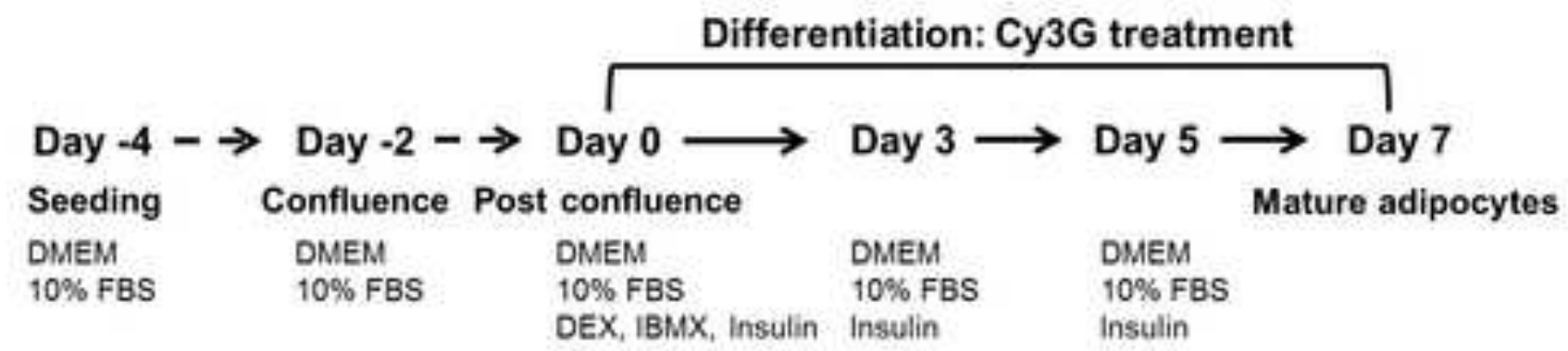

B

C
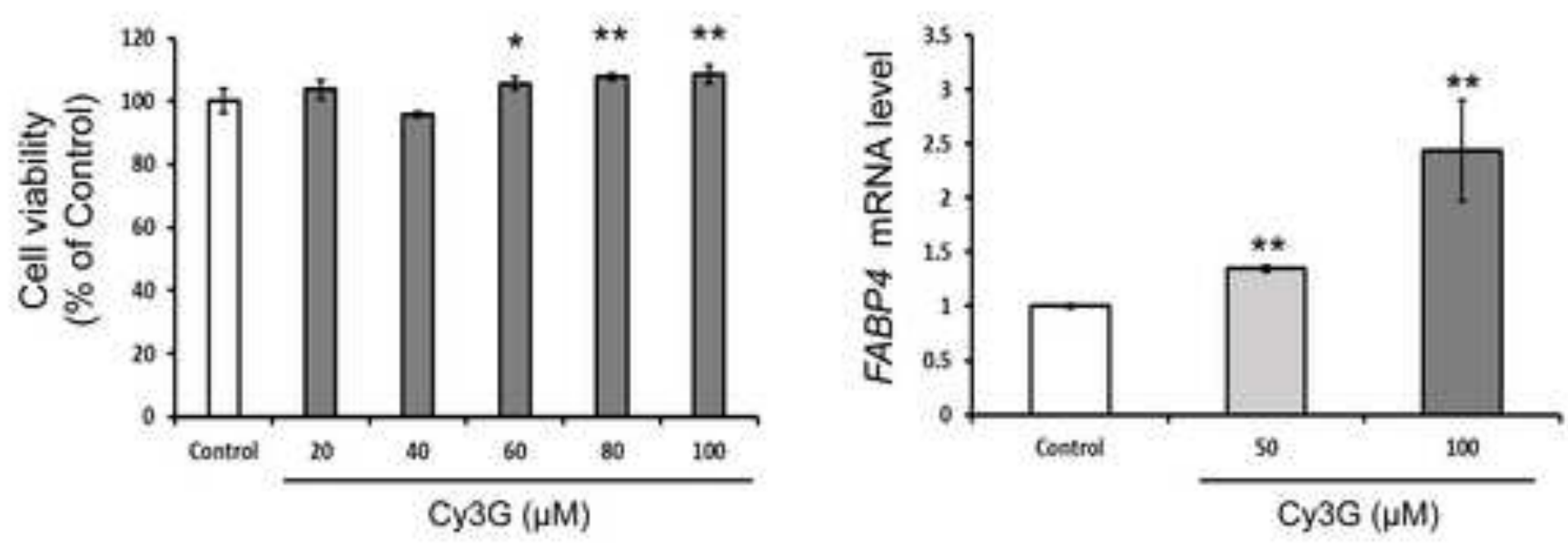

D

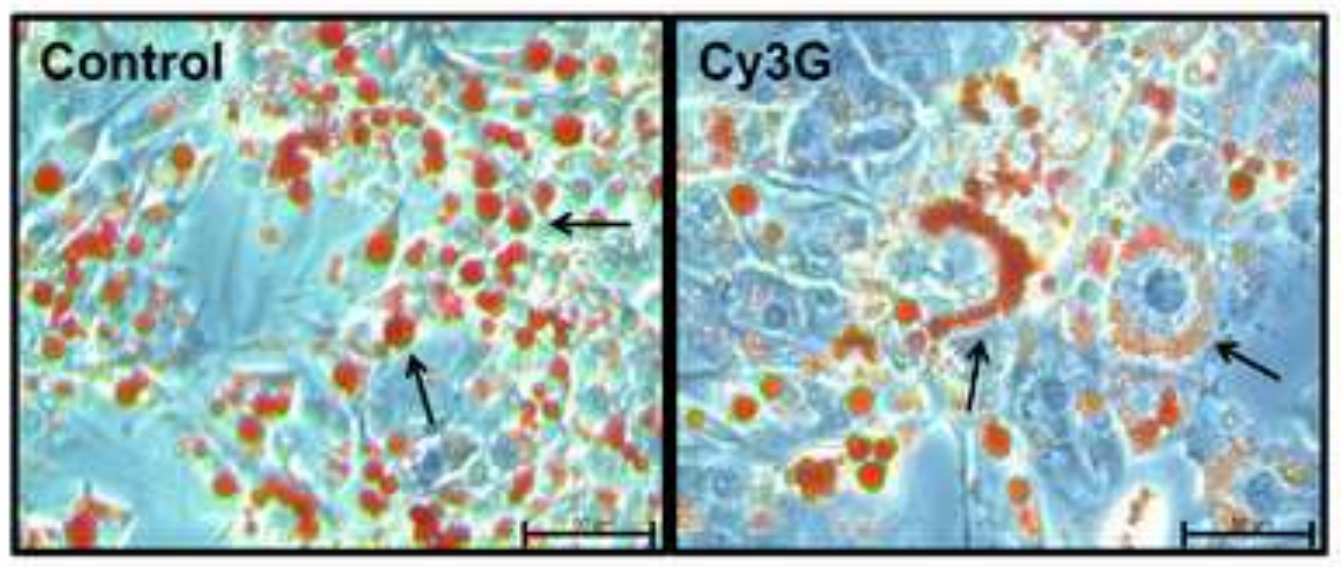

Figure1. 
A
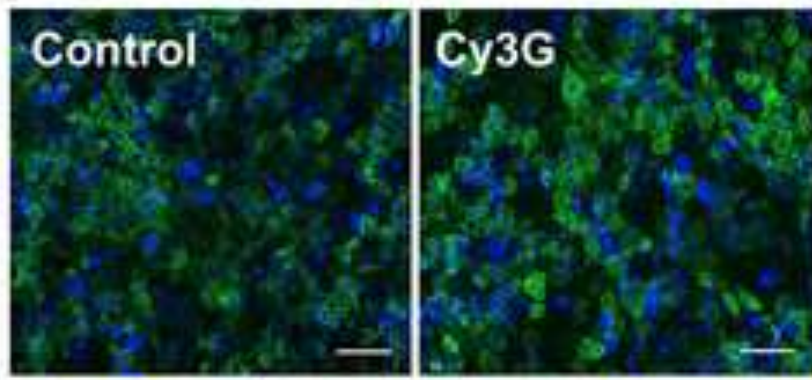

C

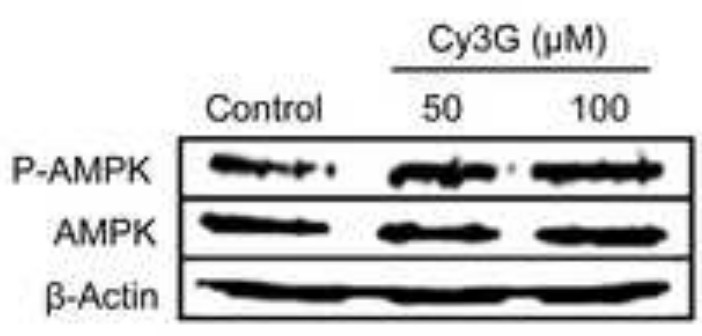

D

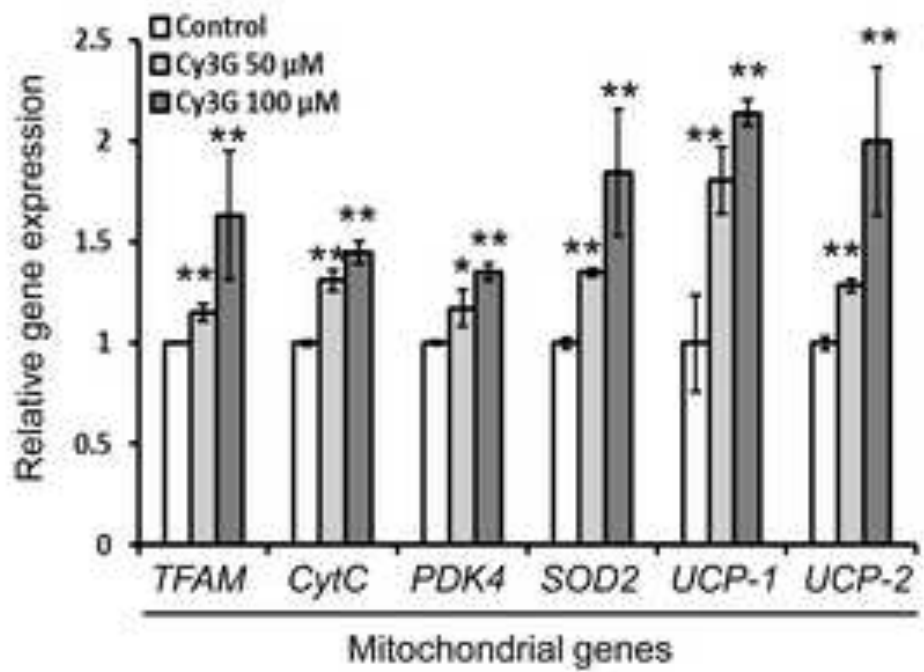

Figure 2.

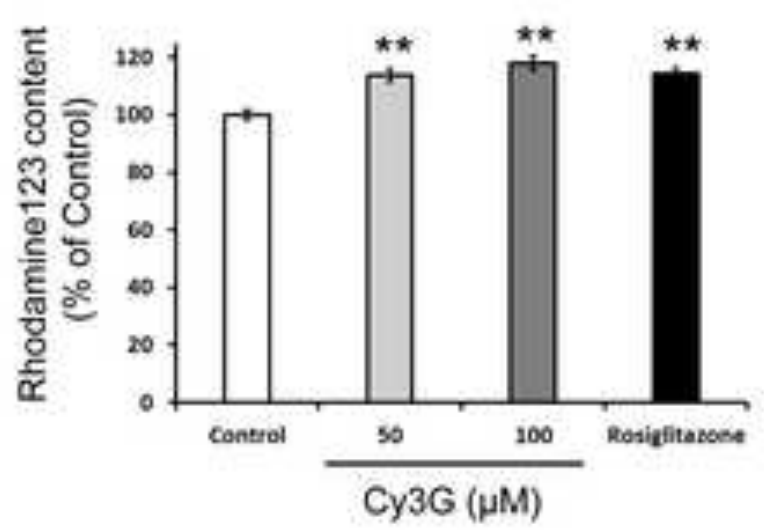

B

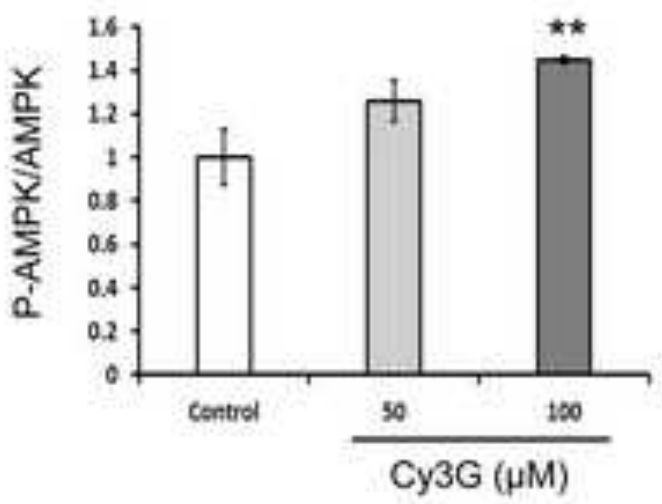

E

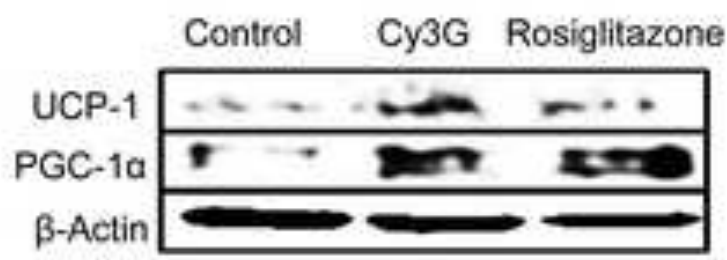

Mitochondrial genes 
A

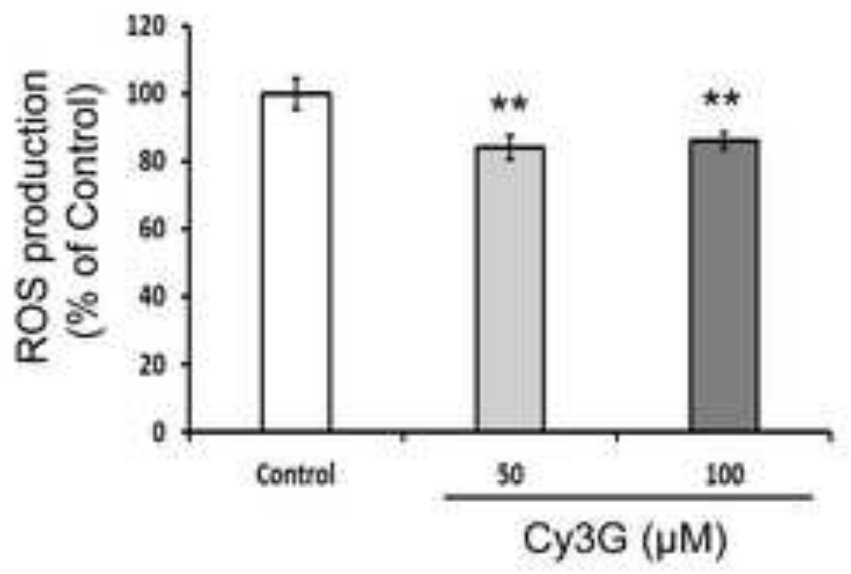

C

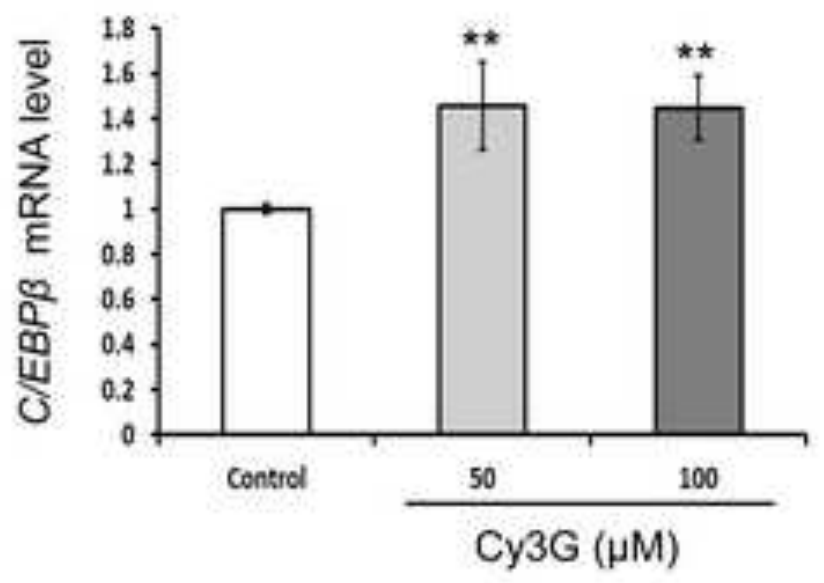

B

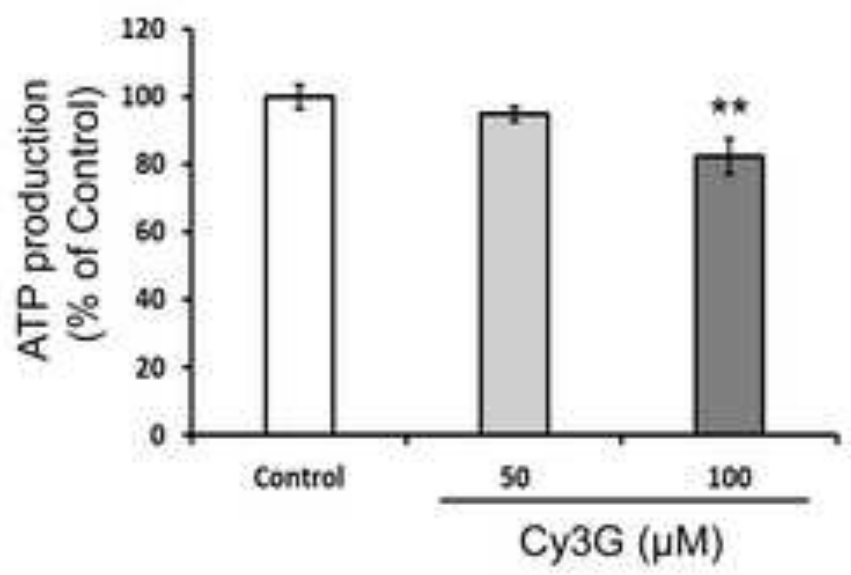

D

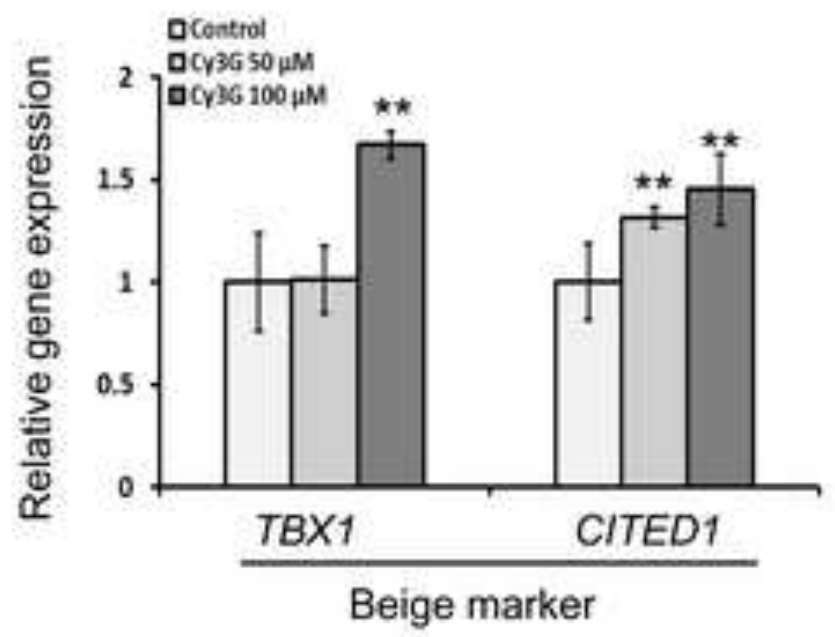

Figure 3. 
A

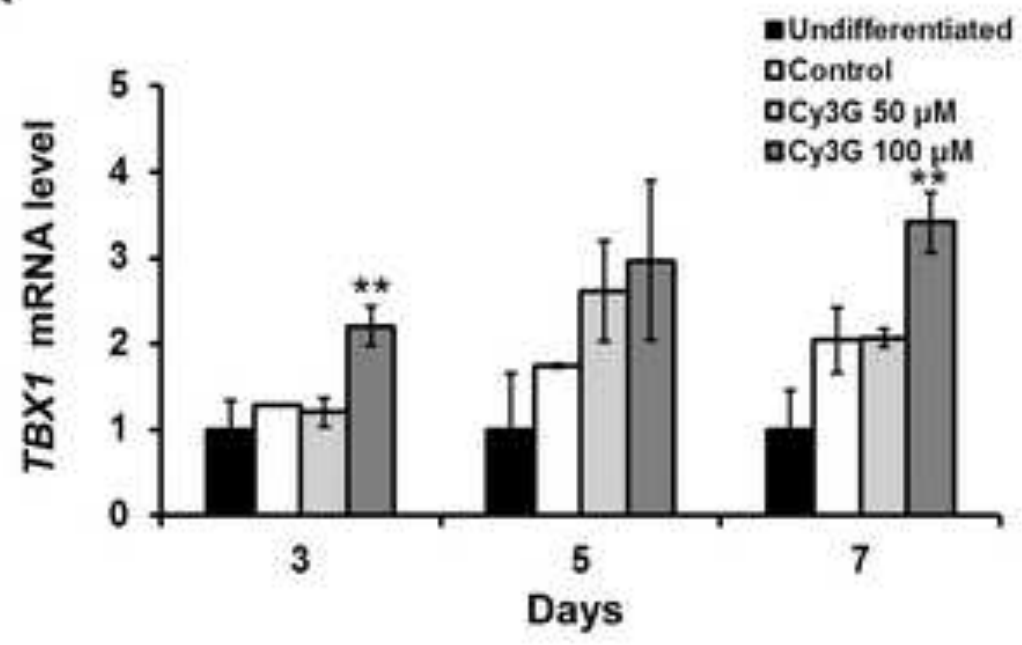

B

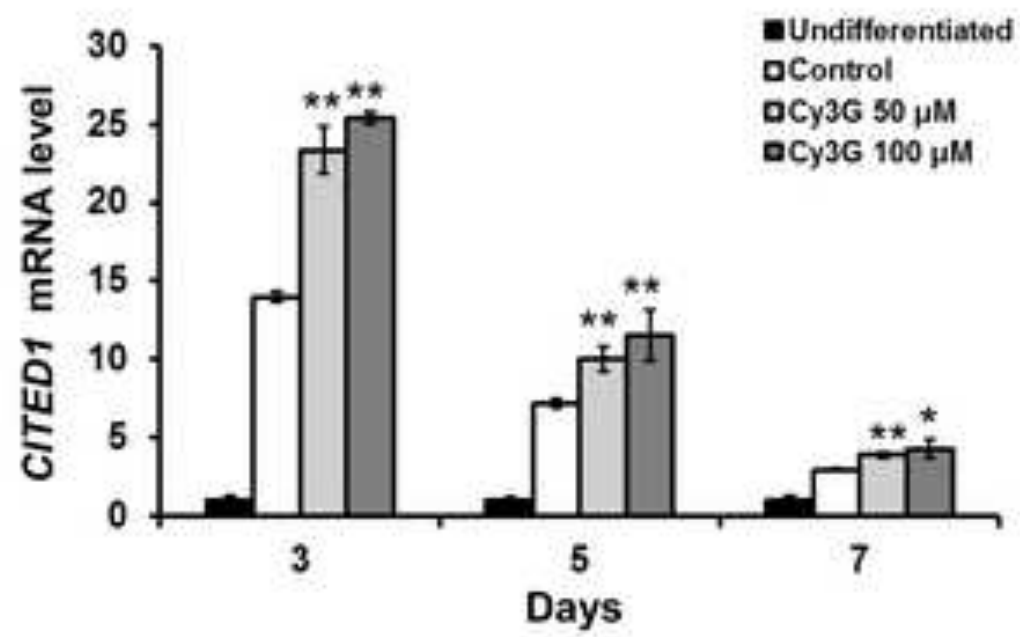

Figure 4. 
A

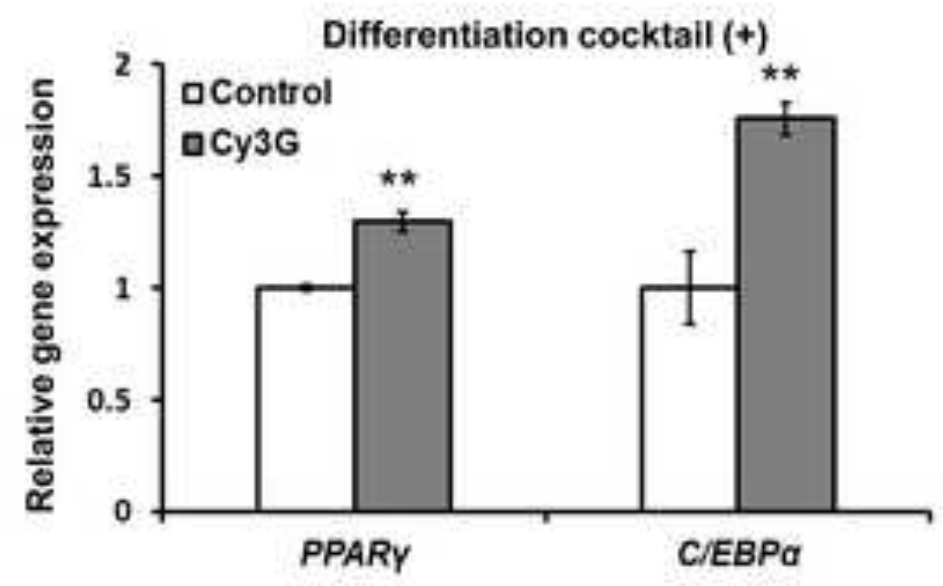

B

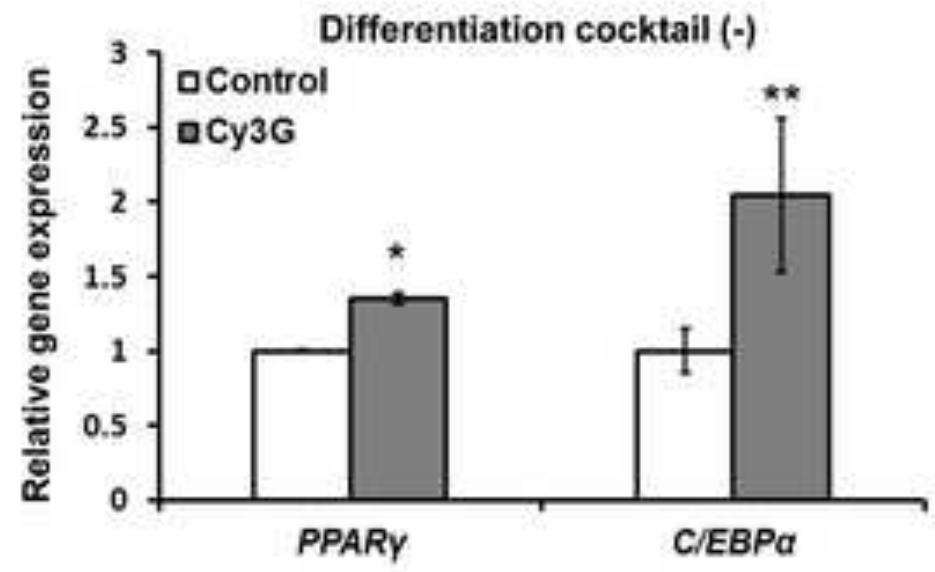

Figure 5. 
A

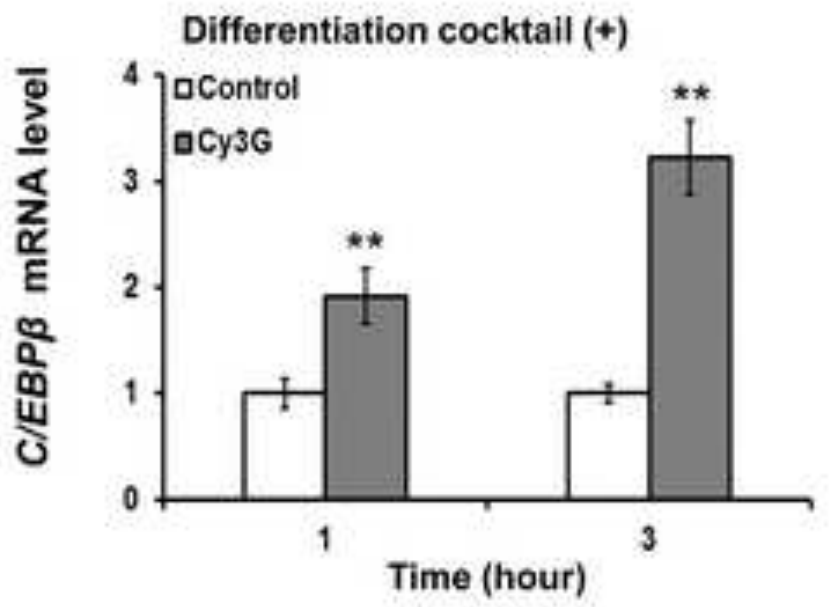

B

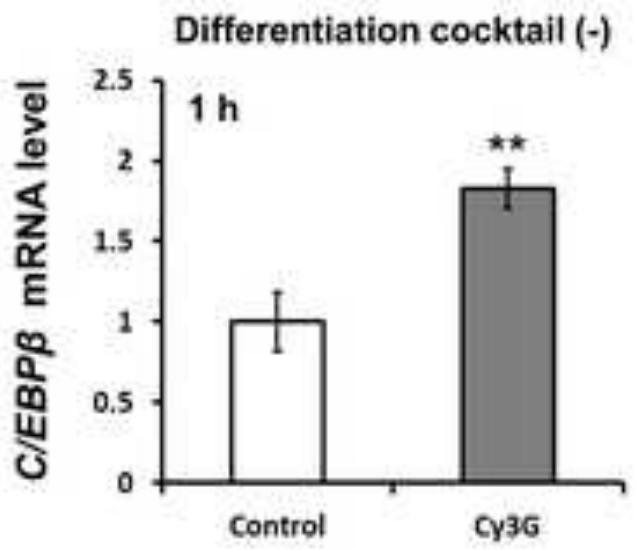

C

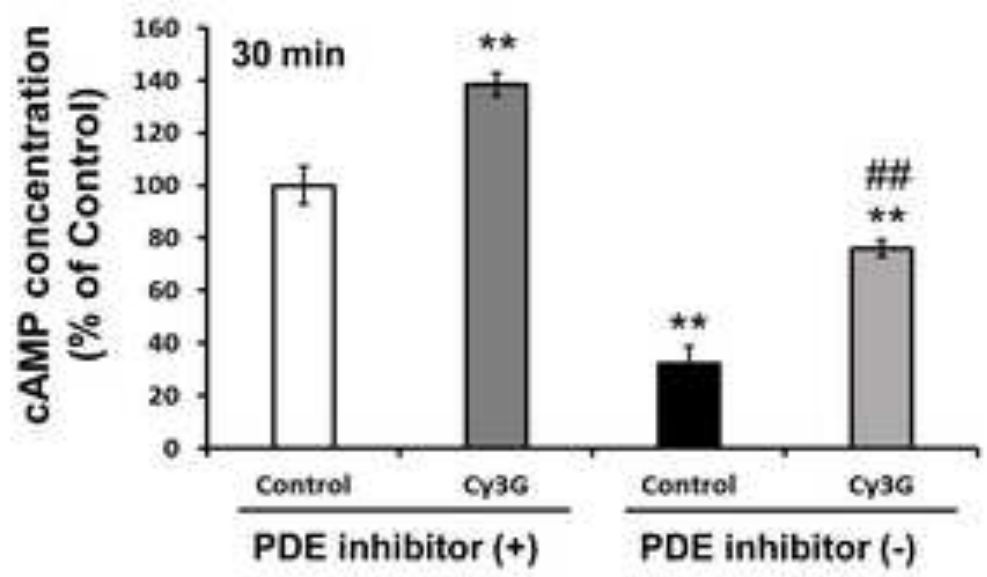

Figure 6. 


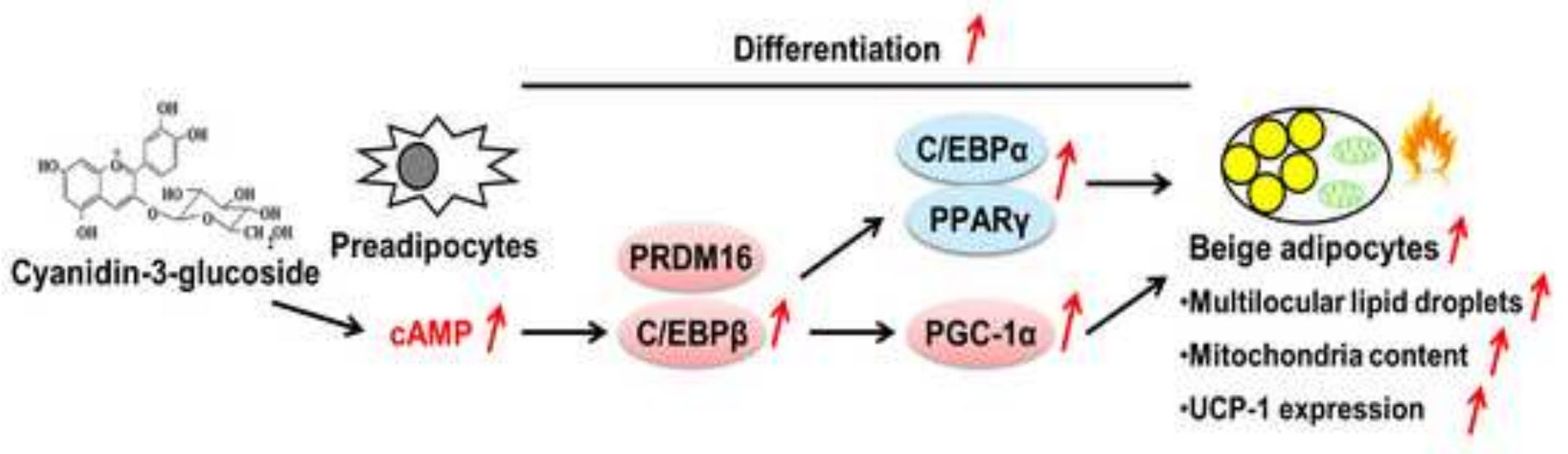

Figure 7.

(




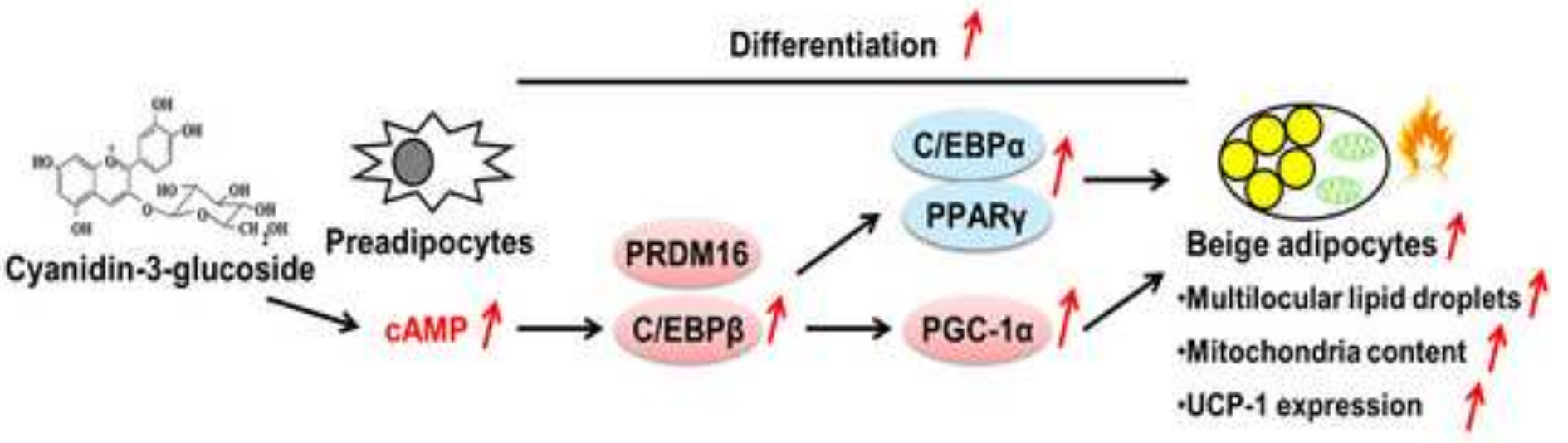

